\title{
Adaptive functions in G0> and YYO earth fault protection
}

\author{
Józef Lorenc ${ }^{1, *}$, Krzysztof Łowczowski ${ }^{1}$, and Bogdan Staszak $^{1}$ \\ ${ }^{1}$ Poznan University of Technology, Institute of Electric Power Engineering, Piotrowo 3A, 60-965 Poznań, Poland
}

\begin{abstract}
In this paper, the benefits resulting from the application of YY0 and G0> earth fault protection with adaptive functions - limiting the risk of outsiders being shocked and reducing the likelihood of twophase short-circuiting with the ground, are presented. The possibilities of improving the effectiveness of admittance protection by introducing special adaptive functions in control algorithms of station automation and decision algorithms of YY0 and G0> criteria are shown. The rules for using such functions have been described and it has been shown that they have a very significant impact on improvement the effectiveness of protection system. It can be stated that in typical medium voltage networks and solutions of active adaptive functions, good conditions are obtained for the operation of admittance protections even at several times higher resistances in the place of earth fault than when these functions are deactivated or not implemented.
\end{abstract}

\section{Introduction}

Currently, the admittance criteria are commonly used in earth fault protections $[1,2,3]$, in which the decision parameter is the admittance described as quotient of the zero sequence components of current and network voltage or active components (conductivity) and passive components (susceptance) of the specified admittance. Such protection devices are sensitive to resistance short circuits whose values in the fault location do not exceed $3000 \Omega$. Practice in recent years shows that there are short circuits with resistances significantly greater than 3000 ohms. It is estimated that in Polish medium voltage networks, the number of such disturbances can account for about $5 \%$ of all ground faults, and these constitute about $80 \%$ of all disturbances in MV grids. These shortcircuits can remain on the network for several hours or more, generating a very strong shock hazard for people and animals around the damaged area.

The limits of the admittance protection sensitivity result inter alia from the setting conditions of the voltage criterion

$$
U_{0 p}>=U_{0 n}
$$

where:

$$
\begin{gathered}
\underline{U}_{0 p}=\frac{E_{p h}}{1+R_{F} \omega C_{0 S}\left(d_{0}+j s\right)} \\
d_{0}=\frac{G_{0 S}}{\omega C_{0 S}} \\
s=\frac{1}{\omega^{2} L_{d} C_{0 S}}-1
\end{gathered}
$$

where:

$U_{0 p}$ - zero sequence voltage component of the measurement voltage,
$E_{p h}$-phase power source voltage,

$R_{F}$ - resistance in the fault location,

$C_{0 s}$ - ground capacity of the network,

$\omega$ - network pulsation,

$d_{0}$ - damping coefficient of network suppression, defined by the quotient of the earth conductance of the $G_{0 S}$ network to its susceptance in $C_{0 S}$,

$s$ - coefficient of earth fault compensation,

$L_{d}$ - inductance of the compensating choke (Petersen coil).

By analyzing formulas (1) and (2), it is easy to show that the voltage sensitivity of the protection can be improved by affecting the values of coefficients $d_{0}$ and $s$ or by lowering the setting value $U_{0 n}$ under certain shortcircuit conditions. However, the decision to use such options should be made only during the earth fault and after the analysis of the ground fault parameters. For this purpose the control algorithms of the devices for enforcing the additional ground fault current and the decision-making algorithms of the protection should be supplemented with adaptive functions. Paragraph below present a solution that meets the features of such functions for two criteria: conductive $\mathrm{G} 0>$ and comparative-admittance YY0.

\section{Adaptive functions in G0> protection}

The essence of the adaptive function in the G0> protection is the connection of the $U_{0 n}$ and $G_{0 n}$ setting values with the logical information on the effective operation of the active zero sequence current forcing arrangement (ACF) [4]. The adaptive function is active only when following the operation of the ACF device:

$$
\begin{gathered}
G_{0 p}>G_{0 n} \\
U_{0 n}>U_{0 p}>U_{0 \min }
\end{gathered}
$$


and consists in changing both setting values. It manages this algorithm, which determines new $G_{0 \text { nad }}$ and $U_{\text {0nad }}$ values based on the zero-sequence value of $G_{0 p}$ conductance and $U_{0 p}$ voltage measured during fault time. $U_{0 m i n}$ in formula (6) means the smallest value of the zero voltage component for the faultless condition.

The effect of the adaptive function is a significant increase in the detected fault resistances, what is shown in Fig. 1.

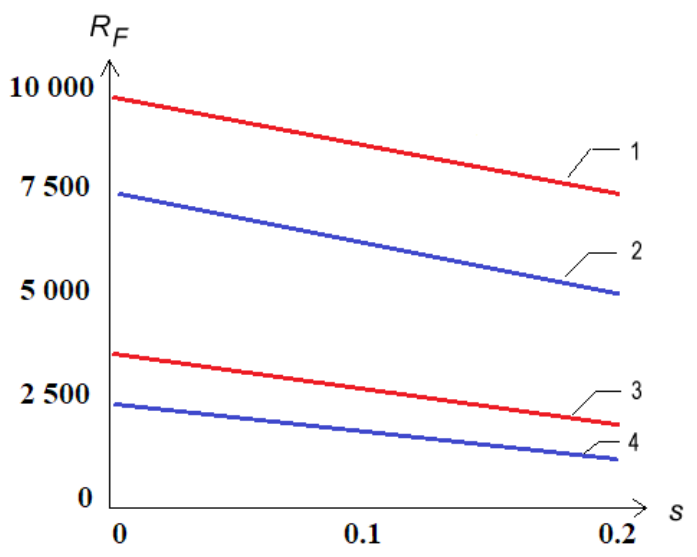

Fig. 1. Effects of protection $\mathrm{G} 0>$ with adaptive function.

Range of detected fault resistance for various values of the earth fault compensation factor $s$ is presented, assuming that $U_{0 \min }$ is equal to 0.05 phase voltage $\left(U_{\mathrm{ph}}\right)$. In Fig. $1 R_{F}$ is shown in ohms, curves 1 and 2 relate to protection with adaptive functions, 3 and 4 without. Curves 1 and 3 apply when the $d_{0}$ coefficient equal to 0.2 , curves 2 and 4 when $d_{0}$ is equal to 0.4 .

\section{Adaptive functions for YYO protection}

The adaptive functions can be implemented in a similar way as in the case of $\mathrm{G} 0>$ protection. Additionally, functionality is extended by selecting the system for enforcing additional active zero sequence current (ACF) or passive current (PCF). During the operation of ACF devices, the coefficient $d_{0}$ is increased, but after replacing the resistor by the reactance the value of the coefficient $s$ changes. In practice, the changes in the coefficient $s$ refer to a value or sign and can be implemented by switching on or off the reactance realizing the automatic operation of the reactive component of the ground fault current (PCF device). The use of the effects of the PCF operation requires the use of a criterion in the $\mathrm{YY} 0$ protection system to investigate the increase in susceptance in the zero-sequence components of the line. Past experience indicates that PCF systems can in many cases be more effective for the needs of earth fault protection than ACF. This applies first of all to earth faults, which are accompanied by significant resistances of the transition at the fault location.

The analysis of the effectiveness of the YY0 protection in $15 \mathrm{kV}$ compensated network with total earth fault capacitive current equals to $120 \mathrm{~A}$ after operation of ACF/PSF forcing devices are given below. Share coefficient of capacitive earth fault of protected line in current of the total network is equal to $20 \%$ and it was assumed that the control value $U_{0 n}$ voltage criterion is $15 \%$ network phase voltage. It was also assumed that the network is symmetrical in the range of phase earth capacities, and the natural attenuation of the earth circuit $d_{0}$ does not exceed 0.04 .

The maximum efficiency (4000 $\Omega$ ) is obtained after the PCF devices operate to the compensating conditions level of 0.1 . However, this effect can only occur if an undercompensated network (detuning $s$ not greater than - 0.1) after operation of PCF goes into a compensating state (coefficient $s$ not greater than 0.1 ) or if overcompensated network (coefficient $s$ not greater than 0.1) after operation of PCF goes into a state of undercompensation (detuning not bigger than -0.1 ).

In other states of compensation, the effectiveness will be reduced and in the case of relatively large detuning factor caused by the operation of PCF devices, the range of detected resistances will be below $2000 \Omega$, which is worse than in the case of ACF devices in a well compensated network.

The adaptive function of the forcing device controller can therefore decide on the type of automation (ACF or $\mathrm{PCF}$ ) and the method of implementing enforcement in the PCF system. The rule should be followed that in the situation of the state of undercompensation the reactance of the coil should be reduced, in case of overcompensation, is increased. An important role in this solution is fulfilled by the control system, in which the voltage level $U_{0}$ is measured and the condition of earth fault compensation is controlled. There are two modes of the PCF system operation. In the first mode the induction current is increased by switching on additional reactance (ALN), whereas in second mode increasing the capacitive current is increased by switching off this reactance (reducing the induction current). In the latter case, the ALN reactance is a permanent element of the compensation system and is included in the undisturbed network operation mode. The system requires continuous information on the current compensation status. The widespread application of follow-up compensation solutions [1] and stationary network-level control systems [5] fully allow access to such information.

In addition, as in the case of $\mathrm{G} 0>$, the effectiveness of the YY0 protection can be increased by lowering the setting value of zero sequence voltage.

\section{References}

1. J. Lorenc, A. Rakowska, B. Staszak, Prz. Elektrotech. 4 (2007)

2. A. Wahlroos, J. Altonen, ABB library (2011)

3. A. Wahlroos, J. Altonen, 12th IET International Conference on Developments in Power System Protection (2014)

4. J. Lorenc, B. Staszak, A. Wiśniewski, Patent PL No 226282 (2017)

5. J. Lorenc, M. Torbus, B. Staszak, Wiad. Elektrotech. 12, (2013) 